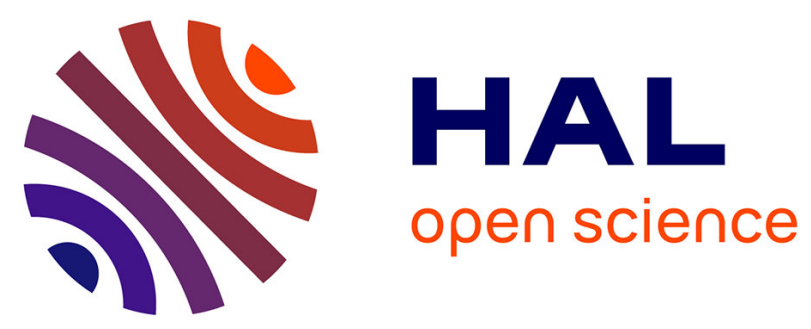

\title{
Influence de la teneur en oxygène sur le comportement au fluage d'alliages $\mathrm{Ti}-48 \mathrm{Al}$
}

P. Hitier, J. Bonnentien, C. Bertrand, M. Cornet, J. Bigot

\section{To cite this version:}

P. Hitier, J. Bonnentien, C. Bertrand, M. Cornet, J. Bigot. Influence de la teneur en oxygène sur le comportement au fluage d'alliages Ti-48Al. Journal de Physique IV Proceedings, 1996, 06 (C2), pp.C2-159-C2-164. 10.1051/jp4:1996222 . jpa-00254200

\section{HAL Id: jpa-00254200 https://hal.science/jpa-00254200}

Submitted on 1 Jan 1996

HAL is a multi-disciplinary open access archive for the deposit and dissemination of scientific research documents, whether they are published or not. The documents may come from teaching and research institutions in France or abroad, or from public or private research centers.
L'archive ouverte pluridisciplinaire HAL, est destinée au dépôt et à la diffusion de documents scientifiques de niveau recherche, publiés ou non, émanant des établissements d'enseignement et de recherche français ou étrangers, des laboratoires publics ou privés. 


\title{
Influence de la teneur en oxygène sur le comportement au fluage d'alliages Ti-48Al
}

\author{
P. Hitier, J.L. Bonnentien, C. Bertrand, M. Cornet et J. Bigot
}

Centre d'Etudes de Chimie Métallurgique, CNRS, 15 rue Georges Urbain, 94407 Vitry-sur-Seine cedex, France

\begin{abstract}
The influence of oxygen on microstructure and creep behaviour of Ti-48Al alloys is investigated. The metallurgical structure of the samples is essentially lamellar in spite of the appearance of small grains at the grain boundaries for the high purity alloy. Therefore, the presence of oxygen allows a stabilisation of the lamellar phase. Micro-hardness does not seem to depend on the content of oxygen but rather on the lamellar nature of these alloys. On the other hand, the presence of oxygen promotes creep resistance, whereas that of $\mathrm{Mn}$ and $\mathrm{Nb}$ reduces the extent of the secondary creep stage.
\end{abstract}

\section{INTRODUCTION}

Les aluminiures de titane sont actuellement considérés comme des matériaux de plus en plus attractifs pour l'aéronautique et l'automobile en raison de leurs propriétés mécaniques et de leur résistance à l'oxydation à hautes températures. Un large domaine de concentration en aluminium a été étudié et les résultats suggèrent que les alliages à 48 at \% d'Al possèdent les propriétés mécaniques optimales [1]. Pour cette concentration d'aluminium, différentes microstructures peuvent apparaître suivant la température du traitement thermique. Les plus importantes sont :

a) la structure entièrement transformée composée de grains à structure lamellaire $\alpha_{2}+\gamma$

b) la structure équiaxe $\gamma$

c) la structure duplex qui comprend des grains lamellaires et des grains $\gamma$.

Les propriétés mécaniques de ces alliages paraissent dépendre fortement de la microstructure [2] : la microstructure lamellaire possède une bonne résistance au fluage alors que la microstructure équiaxe présente une meilleure ductilité. Le but de cette étude est de voir quel est le rôle de l'oxygène sur l'évolution des différentes structures et sur les caractéristiques mécaniques.

\section{TECHNIQUES EXPÉRIMENTALES}

Trois alliages du type $\mathrm{TiAl}_{48}$ d'origines différentes ont été étudiés : un alliage de haute pureté, un alliage de purcté commerciale et un alliage de composition industrielle $\mathrm{TiAl}_{48} \mathrm{Mn}_{2} \mathrm{Nb}_{2}$. Ces alliages 
seront nommés respectivement $\mathrm{A}, \mathrm{B}$, et $\mathrm{C}$ dans le reste de l'article. Ces compositions se situent à la limite du domaine $\gamma$ et du domaine $\alpha_{2}+\gamma$ selon le diagramme d'équilibre binaire proposé par Mc Cullough (3).

Les alliages binaires sont préparés par fusion en lévitation sous atmosphère d'hélium dont la pureté est contrôlée par chromatographic en phase gazeuse, puis coulés dans un creuset en cuivre massif. La fusion se fait sans contamination [4] mais le refroidissement brutal entraîne la formation de fissures. Dans une seconde étape les alliages sont fondus sous la forme de barreaux d'un centimètre de diamètre, dans une nacelle de cuivre refroidi sous atmosphère d'hélium contrôlée. Les barreaux subissent ensuite une fusion de zone, l'inducteur se déplaçant à une vitesse de $120 \mathrm{~mm} / \mathrm{h}$. Le traitement thermique est donc identique pour les trois alliages et ceux-ci ne présentent pas de fissure.

L'alliage A est élaboré à partir de titane de haute pureté [5] préparé par le procédé Van Arkel, dont les principales impuretés sont : $\mathrm{O}=27, \mathrm{Fe}=63, \mathrm{Cr}=14, \mathrm{Ni}=14, \mathrm{Cu}=6,7 \mathrm{ppm}$. L'aluminium a une teneur totale en impuretés inférieure au ppm. L'alliage $\mathrm{B}$ est produit à partir de titane commercial $(\mathrm{O}=1500, \mathrm{Fe}=320, \mathrm{Si}<100 \mathrm{ppm})$ et d'aluminium courant $(\mathrm{Fe}=3,3, \mathrm{Si}=120, \mathrm{Zn}=7$, $\mathrm{Cu}=2,95 \mathrm{ppm})[6]$.

Les microstructures ont été observées sur un microscope optique REICHERT après un polissage mécanique jusqu'à l'alumine $24 \mathrm{H}$ suivi d'une attaque chimique $\left(0,75 \% \mathrm{HF}+1,75 \% \mathrm{HCl}+97,5 \% \mathrm{H}_{2} \mathrm{O}\right)$.

Les mesures de microdureté ont été effectuées à la température ambiante, avec un microduromètre DURIMET sous une charge de $300 \mathrm{~g}$. Chaque résultat est moyenné sur 10 mesures. Les échantillons ont été attaqués par le même réactif que pour l'observation micrographique afin de pouvoir mesurer la dureté de plages bien identifiées.

Les essais de compression ont été effectués à température ambiante sur une machine INSTRON 6025 pilotée par un Hewlett Packard. Les éprouvettes, des cylindres de $3 \mathrm{~mm}$ de diamètre sur $6 \mathrm{~mm}$ de long, ont été mises en forme par usinage du barreau. Cette méthode permet de s'affranchir des différences de structure entre la partie périphérique du barreau au contact de la nacelle refroidie et la partie centrale du barreau.

Les essais de fluage ont été réalisés sur une machine INSTRON TTDML montée en compression pilotée par un Macintosh dont le programme a été modifié pour servir une machine de fluage. Les éprouvettes de fluage, des cylindres de $15,9 \mathrm{~mm}^{2}$ de section sur $9 \mathrm{~mm}$ de long, sont obtenues par la même méthode que les éprouvettes de compression.

\section{RÉSULTATS}

\subsection{Aspect micrographique}

Pour les trois alliages, la structure métallurgique obtenue est essentiellement lamellaire ce qui est caractéristique de ces alliages. (Figure. 1). La taille de grain des barreaux varie de la partie inférieure à la partie supérieure. Pour les trois alliages, dans la partie supérieure les grains possèdent une taille d'environ $500 \mathrm{~mm}$ le long de la direction de solidification. Dans la partie inférieure cette taille peut atteindre $2 \mathrm{~mm}$. Les grains sont allongés dans la direction de croissance qui fait un angle de $45^{\circ}$ avec l'axe des barreaux. L'orientation relative des lamelles varie d'un grain à l'autre.

Les diagrammes de rayons $\mathrm{X}$ montrent que les alliages sont majoritairement de la phase $\gamma$ avec une faible proportion d' $\alpha_{2}$. Dans chaque grain, il y a apparition d'un réseau sombre entourant des zones claires. Ces deux zones couvrent à peu près la même surface et dans les alliages A et $\mathrm{B}$, ces deux zones sont d'environ $70 \mu \mathrm{m}$. En revanche dans l'alliage C (Figure 2) les zones sombres sont plus petites, de quelques $\mu \mathrm{m}$. De telles zones ont déjà ćté observées par Takeyama [7].

D'autre part, au niveau de certains joints de grains, il y a croissance de petits grains probablement de structure $\gamma$. On remarque un plus grand nombre de ces petits grains dans l'alliage de haute pureté. 


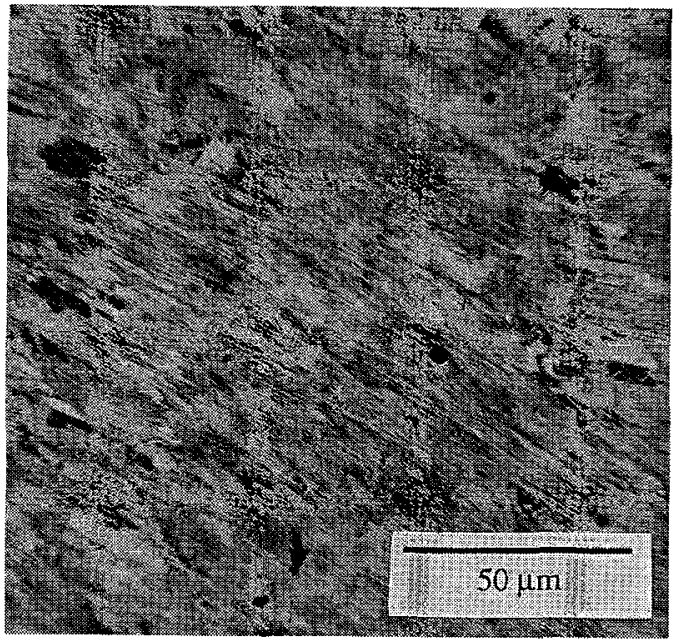

Figure 1. Structure lamellaire de l'alliage de haute pureté après fusion de zone.

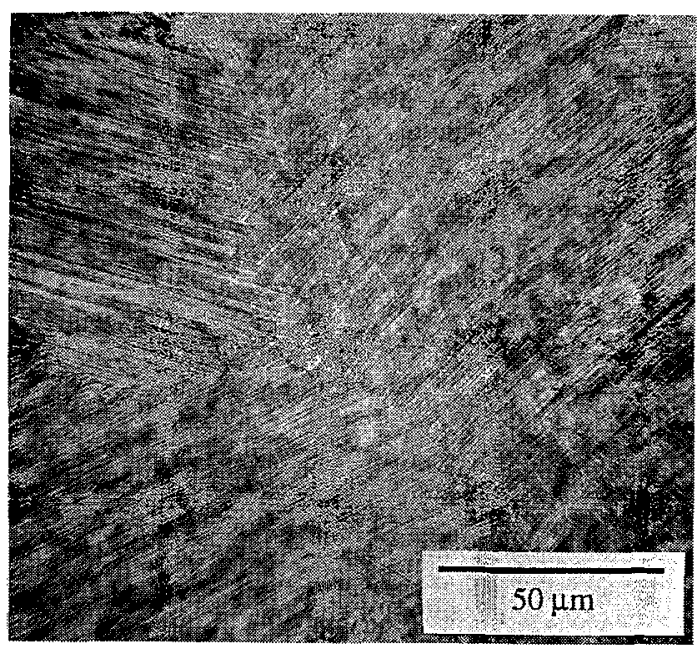

Figure 2. Structure lamellaire de l'alliage industriel.

\subsection{Microdureté et limite élastique}

Les résultats obtenus sont reportés dans le tableau suivant :

\begin{tabular}{|c|c|c|c|}
\hline & $\begin{array}{c}\text { DURETÉ VICKERS } \\
\text { PARTIE CLAIRE } \\
\text { (HV) }\end{array}$ & $\begin{array}{c}\text { DURETÉ VICKERS } \\
\text { PARTIE SOMBRE } \\
\text { (HV) }\end{array}$ & $\begin{array}{c}\text { LIMITE ÉLASTIQUE } \\
\text { 0.2\% MPa }\end{array}$ \\
\hline $\begin{array}{c}\text { Ti-48AI } \\
\text { Haute pureté }\end{array}$ & $350 \pm 20$ & $260 \pm 20$ & $305-335-405-350$ \\
\hline $\begin{array}{c}\text { Ti-48Al } \\
\text { pureté commerciale }\end{array}$ & $340 \pm 30$ & $275 \pm 10$ & $325-330-420-360$ \\
\hline Tí48Al $_{48} \mathbf{M n}_{\mathbf{2}} \mathbf{N b} \mathbf{2}$ & $305 \pm 20$ & 310 \\
\hline
\end{tabular}

Tableau 1. Dureté Vickers et limite élastique en compression des différents alliages brut de fusion de zone fondue.

Comme le montre le tableau 1, la microdureté des échantillons dépend de la zone que l'on étudie dans les grains. En effet si l'on mesure dans les parties claires, on obtient une dureté similaire pour l'alliage A et l'alliage B $(350 \pm 20 \mathrm{HV}$ pour l'alliage de haute pureté et $340 \pm 30 \mathrm{HV}$ pour l'alliage de pureté commerciale). En revanche, si l'on mesure dans le réseau sombre, la dureté n'est plus que de 
$260 \pm 20 \mathrm{HV}$ pour l'alliage A et de $275 \pm 10 \mathrm{HV}$ pour l'alliage B. Pour l'alliage C, les parties sombres et claires n'étaient pas assez larges pour que l'on puisse avoir une empreinte sur une seule zone. On ne pouvait donc pas séparer les duretés des deux zones. On n'a donc obtenu qu'une valeur moyenne de $305 \pm 20 \mathrm{HV}$. On remarque que cette valeur correspond à la moyenne des valeurs obtenues pour les deux autres alliages. Comme les deux zones sont à peu près en mêtme quantité dans chaque grain, on peut supposer que la dureté des deux zones différentes est la même que pour les deux autres alliages.

\subsection{Fluage}

Les essais ont été effectués en compression avec une contrainte de $260 \mathrm{MPa}$, sous un vide de $10^{-4} \mathrm{~Pa}$, dans un four à $802^{\circ} \mathrm{C}$. La température de fusion des alliages $\mathrm{Ti}-48 \mathrm{Al}$ étant de $1773 \mathrm{~K}$, nous nous trouvons dans le cas du fluage restauration. La courbe caractéristique de ce fluage possède trois stades distincts [8]. Durant le premier stade, correspondant au fluage transitoire ou parabolique, la vitesse de fluage décroît en $t^{-n}$. Durant le second stade ou fluage stationnaire, la vitesse de fluage reste constante et est caractéristique du matériau. Enfin durant le troisième stade ou fluage accéléré, on a une augmentation de e. Les trois courbes $\varepsilon=\mathrm{f}(\mathrm{t})$ ont une caractéristique en commun : la durée de leur stade primaire est faible. Les vitesses de fluage stationnaire dépendent de la pureté de l'alliage. En effet l'alliage A possède une vitesse dix fois plus grande $\left(210^{-6} \mathrm{~s}^{-}\right)$que celle des deux autres alliages $\left(1,6310^{-7} \mathrm{~s}^{-1}\right.$ pour l'alliage $B$ et $1,5110^{-6} \mathrm{~s}^{-1}$ pour l'alliage C) (Figure 3 ). D'autre part la durée du stade secondaire de l'alliage de haute pureté est nettement inférieure à celle des deux autres alliages. On remarque aussi que la durée du stade secondaire est environ deux fois plus élevée dans le cas de l'alliage B que dans le cas de l'alliage C. Pour les trois échantillons, l'apparition de petits grains au cours du fluage confirme bien l'existence d'une recristallisation dynamique $[9,10]$.

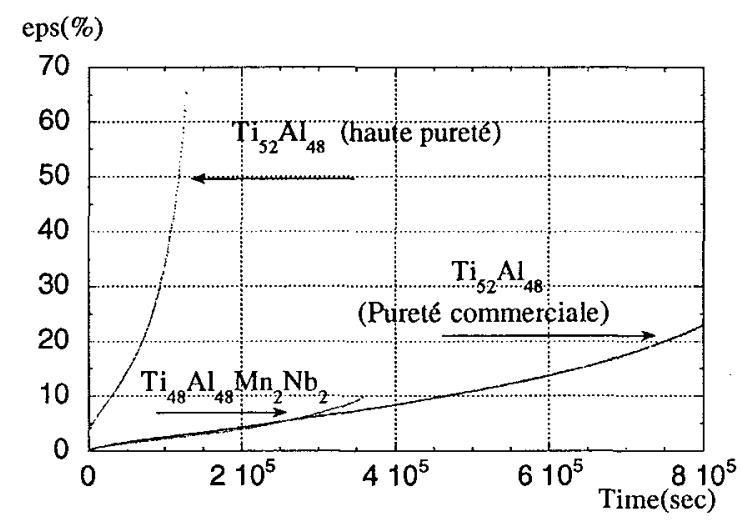

Figure 3. Courbe de fluage des trois alliages étudiés.

\section{DISCUSSION}

\subsection{Effets de l'oxygène sur la microstructure}

Lors du refroidissement, et selon le diagramme d'équilibre [3] le premier cristal obtenu est probablement de structure $\beta$, le déplacement du front de solidification devant conduire à la formation 
d'une structure colonnaire de phase $\beta$. Du fait du déplacement, la température diminue et la transformation péritectique $\beta+$ liq $\rightarrow \alpha+$ liq aboutit à la formation de $\alpha$. Le refroidissement étant lent, les grains de phase $\alpha$ se transforment selon une structure lamellaire biphasée $\alpha \rightarrow \alpha+\gamma$ selon le mécanisme proposé par Y.Yamabé [11]. A plus basse température la phase $\alpha$ s'ordonne pour donner $\alpha_{2}$.

$\mathrm{Au}$ cours de la fusion de zone le gradient de température qui suit le front de solidification se déplace à une vitesse telle que les cinétiques de transformation ne permettent pas une évolution très sensible de la structure lamellaire. Toutefois, dans l'alliage de haute pureté, l'apparition de petits cristaux aux joints de grains semble indiquer qu'en l'absence d'oxygène l'évolution structurale des alliages est plus importante. L'oxygène stabiliserait la phase lamellaire. L'addition de manganèse et de niobium, en revanche, n'entraîne pas de différence notable au niveau de la structure proprement dite ; elle permet cependant de réduire la taille des zones claires et sombres. L'existence de cette répartition pourrait être liée à une hétérogénéité chimique.

\subsection{Effets de l'oxygène sur la microdureté}

La dureté des différents échantillons ayant une structure lamellaire est sensiblement identique pour les trois alliages bien que leur concentration en oxygène diffère. Ceci implique que la microdureté dépende essentiellement de la microstructure de l'alliage et peu de la teneur en oxygène. De même l'addition de manganèse et de niobium ne paraît pas améliorer cette caractéristique mécanique. Les valeurs de limite élastique relevées sur les courbes de compression varient dans un large domaine et ne semblent pas très influencées par la pureté.

\subsection{Effets de l'oxygène sur le comportement au fluage}

L'oxygène semble avoir un rôle important sur le comportement au fluage de ces alliages. En effet la vitesse de fluage stationnaire est beaucoup plus élevée pour l'alliage de haute pureté que pour les deux autres alliages. Ceci est peut-être lié à la présence de cristaux $\gamma$ dans les joints de grains. Cependant pour la température d'essais la déformation aux joints de grains ne doit pas être importante. Cette différence de comportement peut être reliée à la faible teneur en oxygène de la phase $\alpha_{2}$ qui piège généralement l'oxygène [12] et qui de ce fait serait plus ductile. L'oxygène semble donc améliorer la résistance au fluage des alliages TiAl48.

Par ailleurs la présence des deux éléments d'addition, manganèse et niobium, ne modifie pas la vitesse de fluage, mais elle diminue la durée du stade secondaire.

\section{CONCLUSION}

L'aspect micrographique ainsi que la microdureté et la résistance au fluage à $800^{\circ} \mathrm{C}$ ont été étudiés sur des alliages du type TiAl 48 de différentes puretés, bruts de fusion de zone.

Du point de vue micrographique, la présence d'oxygène semble stabiliser la phase lamellaire tandis que l'ajout de niobium et de manganèse ne modifie pas sensiblement cette structure mais pourrait modifier l'hétérogénéité chimique des alliages.

La microdureté et la limite élastique à la température ambiante dépendent essentiellement du caractère lamellaire de la structure. Les variations de la teneur en oxygène ou de celle des éléments d'addition ne paraissent pas avoir d'influence En revanche les variations locales de microdureté pourraient être associées à l'hétérogénéité chimique du matériau. 
$\mathrm{Au}$ contraire, pour ce même état structural, la présence d'oxygène favorise la résistance au fluage alors que les additions de niobium et de manganèse réduisent l'étendue du fluage secondaire.

\section{REFERENCES}

[1] Huang S.-C. and Shih D. S., Micro ed. Y.-W.Kim and R.P. Boyer. TMS, Warrendale, PA, (1991), 105-122.

[2] Huang S.-C, Metall. Trans. 23A (1992 .375-37.7

[3] McCullough C., Valencia J.J. ,Levi C.G. and Mehrabian R., Acta Metall. 37 (1989), 1321-1336.

[4] Matyka J., Faudot F. and Bigot J., Fourth International Conference on Titanium, Kyoto (1980) 2942.

[5] Bigot J., Colloque Europeen de métallurgie sous vide, Le Vide 177 (1975) 151.

[6] Dimitrov C., Pastol J.-L., Bigot J. and Dimitrov O., Journal de Physique III vol 3 (1993) $481-485$.

[7] Takeyama M., Mater. Sci. Eng. A 152 .(1992) p 383.

[8] Bénard J., Michel A., Philibert J. et Talbot J., Métallurgie générale, (Masson \& Cie, 1969).

[9] Bartels A., Seeger J. and Mecking H., Mat. Res. Soc 288 (1993) pp. 1179-1184.

[10] Hayes R.W. and McQuay P.A., Scr. Metall. Mater. 30 (1994) pp. 259-264.

[11] Yamabe Y., Takeyama M., Kikuchi M., Proceeding of the International Conference of Gamma Titanium Aluminides, TMS, 1995.(To be published).

[12] Denquin A., Naka S., Huguet A. and Menand A., Scripta Metall. et Mater., 28 (1993) p 1131-36. 